\title{
Astronomy in the Crossroads of Interdisciplinary and Multidisciplinary Sciences
}

\author{
Areg M Mickaelian* and Sona V Farmanyan \\ NASRAV Ambartsumian Byurakan Astrophysical Observatory (BAO), Armenia
}

Submission: March 12, 2021; Published: April 07, 2021

*Corresponding author: Areg M Mickaelian, NASRAV Ambartsumian Byurakan Astrophysical Observatory (BAO), Armenia

\begin{abstract}
Interdisciplinary and multidisciplinary sciences over the last few decades have become the major booster of science development. The most important discoveries occur just at the intersection of sciences and in collaboration of several fields. There appeared such intermediate fields as mathematical physics, physical chemistry, biophysics, biochemistry, geophysics, etc. In Astronomy, Astrophysics has long been the main field, and in present Archaeoastronomy, Astrochemistry, Astrobiology, Astroinformatics (which is tightly related to Virtual Observatories) are developing. On the other hand, in recent years many science areas surfeit of research on Earth, more and more use data coming from the Space and are being developed just due to them. It is possible that soon, various science areas create Space departments or simply develop their research in close collaboration with astronomers. Interesting discoveries have been made in studies of astronomical topics in various areas of culture; such topics are widely used in folklore, other genres of literature, painting, and architecture. Astronomy has also a leading role in Scientific Tourism, Scientific Journalism and in general, dissemination of popular science or public outreach.
\end{abstract}

Keywords: Interdisciplinary sciences; Multidisciplinary sciences; Transdisciplinary sciences, Astronomy; Astronomy in culture

\section{Introduction: Current Trends in Astronomy}

\section{Development}

By studying the features of the current stage of development of astronomy, we have distinguished the current tendencies:

i. Broader usage of modern astronomical instruments and research methods (telescopes, various modes of spectroscopy (IFU, MOS, etc.), active and adaptive optics, digital receivers, software).

ii. Multiwavelength studies ( $\gamma$-rays, X-rays, UV, optical, IR, sub-mm, mm, and radio; using space telescopes, etc.) and Multimessenger studies, including the development of neutrino, cosmic rays, and gravitational wave astronomy.

iii. Results based on large-scale astronomical data processing (Astrostatistics, Virtual Observatories, computer modeling, etc.).

iv. Large international astronomical projects and large collaborations (OPTICON, SDSS, ESA Gaia, PanSTARRS, SKA, ESO ELT, LSST, etc.). v. The expansion of connection of Astronomy to other science fields (Astro Particle Physics, Astrochemistry, Astrobiology, close connection with Optics, Mechanics, Spectroscopy, Atomic and Nuclear Physics, Informatics, mathematical modeling, and with other spheres of culture).

In this article we will discuss the last point in more details. If we consider the recently revised divisions of the International Astronomical Union (IAU), we can see that only 4 out of 9 divisions are dedicated to purely astronomical fields and 4 are devoted to interdisciplinary and multidisciplinary sciences, or at least their elements are present (Figure 1): Division B - Equipment, Technology and Data Sciences (related to Optics, Modern Technologies, Astroinformatics, and Virtual Observatories); Division C - Education, Public Relations and Heritage (related to Archaeoastronomy, Astronomy in Culture, Astronomical Education and Public Outreach); Division D - High Energy and Fundamental Physics (related to Nuclear and High Energy Physics); and Division F - Planetary Systems and Biology (related to Astrobiology). 


\section{IAU Divisions}

- A: Fundamental Astronomy

- B: Facilities, Technologies and Data Science

- C: Education, Outreach and Heritage

- D: High energy phenomena and Fundamental Physics

- E: Sun and Heliosphere

- F: Planetary Systems and Bioastronomy

- G: Stars and Stellar Physics

- H: Interstellar Matter and Local Universe

- J: Galaxies and Cosmology

Figure 1: Current IAU Divisions showing the heavy presence of interdisciplinary and multidisciplinary sciences.

Such tendencies in astronomy are also evident by a number of interdisciplinary and multidisciplinary IAU commissions and working groups, UNESCO's "Astronomy and World Heritage" Program, the International Virtual Observatory Alliance (IVOA), regularly held "Astronomical Data Analysis Software and Systems" (ADASS) Conferences, Committee of Space Research (COSPAR), International Council on Monuments and Sites (ICOMOS), the European Society for Astronomy in Culture (SEAC), International Science Council (ISC, formerly ICSU), World Data System (WDS), Information Theories \& Applications (ITHEA) organization, the International Planetary Data Alliance (IPDA) and others. All these organizations were mainly established in the 2000s.

The Relation of Astronomy to other Sciences and Culture

Astronomy can really be considered as a leading link between interdisciplinary and multidisciplinary sciences. The point is that Astronomy, Astrophysics, Astrochemistry, Astrobiology, Archaeoastronomy, Theoretical Astrophysics (the use of Mathematics in Astrophysics), Astrostatistics, Astroinformatics (closely related to Virtual Observatories) have been developed for a long time. On the other hand, in the recent years, many fields of sciences being satisfied with research on the Earth, more and more use the data obtained from the Universe and are developed due to them. It is quite possible that soon different sciences will create Space divisions or simply will develop their research in close collaboration with astronomers. Interesting discoveries have been made in the study of astronomical topics in various fields of culture. Such topics have been widely used in folklore, other genres of literature, painting and architecture. Astronomy also plays a leading role in scientific tourism, scientific journalism, the dissemination of science in general, or the popularization of science (public outreach), which strengthens the connection between science and society.

At the International Year of Astronomy, declared by UNESCO (IYA-2009), the International Astronomical Union developed a ten-year strategic plan for the development of astronomy, according to which the emphasis is especially on the development of close relations between astronomy and other fields of sciences, technology and culture. The IAU has declared its Strategic Plan in 2009, according to which Astronomy will play a key role among all sciences and research, technologies and skills, and cultures and societies. Figure 2 gives the schematic picture of this plan. In frame of this, world Office of Astronomy for Development (OAD) and 11 Regional Offices of Astronomy for Development (ROADs; one in Armenia) have been founded [1]. Below we give examples of relation between astronomy and a number of other sciences, which prove the important role of astronomy in the development of interdisciplinary and multidisciplinary sciences.

\section{Astronomy and Philosophy}

Philosophical categories such as matter, space and time are directly related to the formation and development of forms of energy and matter in the Universe. By studying the origin and development of the Universe in astronomy, we significantly enrich our philosophical understanding. In Philosophy, a subject of centuries-long debates and ideological struggles has also been the discussions on Geocentrism and Heliocentrism and related to them is the religious notion of Anthropocentrism, which has been rejected by astronomical facts. However, the Problem of Extraterrestrial Civilizations (SETI/CETI) has not yet been finally resolved, although we are closer than ever to discovering other civilizations. 


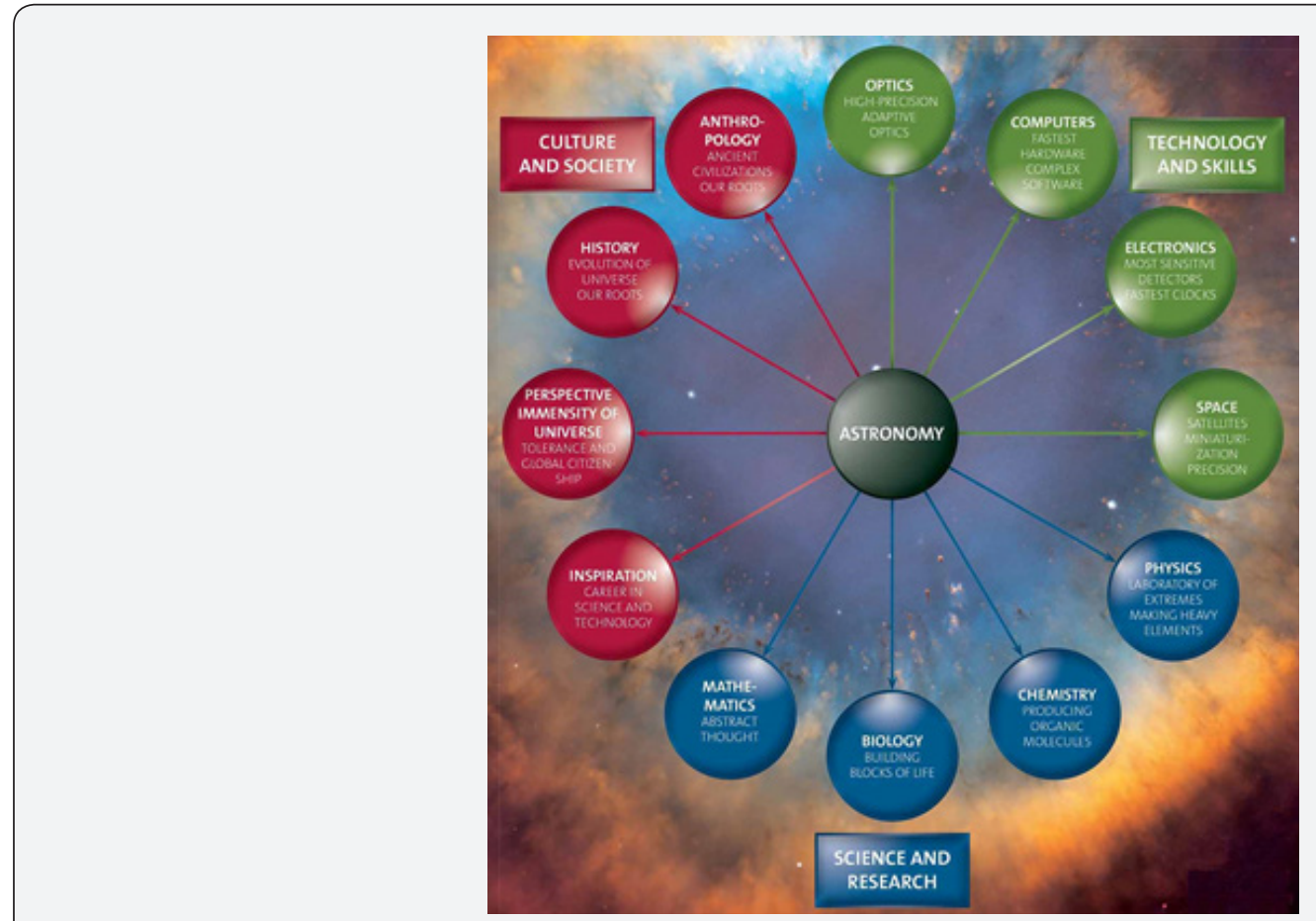

Figure 2: The schematic picture of the IAU Strategic Plan for 2010-2020.

\section{Astronomy and Physics}

Though the modern astronomy (particularly the Byurakan Astrophysical Observatory) is mainly engaged in astrophysical research, but when we look at the sections of astronomy, we are convinced that there are many other branches: Astrometry, Chronology (Calendar and Chronology), Celestial Mechanics, Galactic Astronomy, Planetary Science, Cosmology and Cosmogony, Observational and Theoretical Astronomy, Computational Astronomy, non-optical, neutrino and gravitational wave Astronomy, etc. However, the connection between astronomy and physics plays an important role, which is expressed especially in the fields of Astrophysics, Solar Physics and Astroparticle Physics. On the other hand, almost all the branches of physics - mechanics, optics, electromagnetism, atomic and nuclear physics, the theory of relativity - are used in astronomy and are directly related to the study of the Universe. Moreover, at present they are mainly enriched and developed based on astronomical data, as the Universe is a huge laboratory, where there are all the conditions for Physics "experiments". It is especially worth mentioning the development of atomic and nuclear physics due to the information received from the Space, the testing of the theory of relativity by means of cosmic phenomena. On the other hand, the development of optics is directly related to astronomy since 1609 , from the first telescope made by Galileo to the largest modern optical devices, telescopes with $10 \mathrm{~m}$ and larger in diameter mirrors. It is more interesting to consider all modern physics on a cosmological scale: quantum field theory, theories of unification of forces, formation of elementary particles, nuclear reactions and synthesis of chemical elements, black holes, etc. All these phenomena and theories can be "tested" and understood only within the framework of the "Warm Universe" theory, which is widely used in Cosmology.

\section{Astronomy and Chemistry}

Many of the chemical elements were first discovered in Space and then discovered or synthesized on Earth. The second most common element is Helium, originally in 1868, during the Solar eclipse, was discovered in the spectrum of the Sun by the French astronomer Jules Janssen and the English astronomer Norman Locker. It was named after the Sun (Greek "Helios" - Sun). It was detected only in 1882 on the Earth and obtained a final approval in 1903 at Earth conditions. Chemists and astronomers believe that other chemical elements can be found in the Universe, as well as the chemical composition of the Universe we know is still being revised, which is why Astrochemistry is currently in full swing. A comparative study of the chemical composition of the Universe and its individual systems and cosmic bodies sheds light on the chemical evolution of the Universe. In the above-mentioned "Hot Universe" cosmological theory, the problem of chemical elements, from the simplest to the most complex, the problem of synthesis and changes in their composition plays an important role. Since 1937, numerous molecules have been found in the coldest places 
in Space, mainly in interstellar environments, for a total of 189 , including 43 two-atom ones, 41 three-atom ones, 26 four-atom ones, 19 five-atom, 15 six-atom, 9 seven-atom, 11 eight-atom, 10 nine-atom, and containing ten or more atoms - 15 types of molecules, which also enrich the modern chemistry.

\section{Astronomy and Biology}

Many organic compounds and other molecules necessary for life have been discovered in Space: water, oxygen, carbon, methane, carbon monoxide ice granules, carbon dioxide, formaldehyde, crystalline silicates, di- and tri- acetylene $\mathrm{C}_{4} \mathrm{H}_{2}$ l. $\mathrm{C}_{6} \mathrm{H}_{2}$ ), complex organic molecules (Polycyclic Aromatic Hydrocarbons, $\mathrm{PAH}$ ), which are considered the "building bricks" of life, etc. The connection between Astronomy and Biology is very wide: it includes Space Biology (one of the founders of which is considered to be Norayr Sisakyan) - a science that studies unusual, unique factors in the Universe: weightlessness, Earth's gravity, radiation, ecology of closed systems, the basics of astronauts training and selection, metabolism of organisms, and many other questions, the Problem of Extraterrestrial Civilizations (the study of the possible biological forms of aliens; SETI - Search for Extraterrestrial Intelligence and CETI - Communication with Extraterrestrial Intelligence), the Theory of Cosmic Panspermia (the theory of the possible propagation of life from one planet to another through comets), Astrobiology (which at large unifies all listed areas), Alternative Biology (consideration and study of other than Carbon-Oxygen forms of life), Astrology (in positive sense; influence of cosmic bodies on biological beings living on Earth), influence of astronomical phenomena in paleontology (destruction of dinosaurs by meteorite impact, freezing of the Earth's surface due to disruption of Solar activity cycles, etc.). A detailed review on the Problem of Extraterrestrial Civilizations and Extrasolar Planets is given in Mickaelian [2].

\section{Astronomy and Geology}

The reason for the slow development of Geology is the limitation of the Earth, but in recent years the study of the Moon, other planets of the Solar System and their satellites has led to a significant enrichment of Geology. The fields of Lunar Science and Planetology (Planetary Science) have appeared, including individual fields of Martian Science, Venus Science and Mercury
Science, which are especially developed. In contrast to the relatively flat terrain of the Earth, the mountains of Mars reach a height of $25 \mathrm{~km}$, active formations have been found on the planets and their satellites, in their outer layers there are many interesting "geological" formations, the study of which enriches our knowledge. The field of Comparative Planetology has been created.

\section{Astronomy and Mathematics}

Theoretical astronomy (celestial mechanics, astrodynamics) and theoretical astrophysics (the inner structure of the stars, the theory of radiative transfer, etc.) are based entirely on the study of the cosmic bodies and phenomena by mathematical methods. The most complex equations in mathematics are used in astrophysics. The study of the large-scale structure of the Universe is based on Theoretical Cosmology, which is also the mathematical apparatus of the General Theory of Relativity. Inverse Problems, Integral Equations, Mathematical Modeling, Astrostatistics the Theory of Errors are widely used and developed in astronomy. Due to the huge amount of data accumulated, the role of statistics in astronomy is so great that conferences on "Statistical Problems in Modern Astronomy" and other similar topics are regularly held.

\section{Astronomy and Informatics}

One of the most widely used applications of the Computer Science is Astronomy, which is associated with a vast amount of information in it. At present, this branch is called Astroinformatics, which includes mathematical modeling, Astrostatistics, work with large repositories and databases, Computational Astronomy, Virtual Observatories, etc. Numerous astronomical software and systems have been developed, in particular the All-Sky Virtual Observatory (ASVO). The development of astronomy is particularly facilitated using modern methods in astronomy education, including in the form of computer games, public professional astronomical programs. Regular conferences are held on "Astroinformatics", "Big Data in Astronomy", "Astronomical Data Analysis Software and Systems". We give in Figure 3 a schematic view of Big Data in Astronomy. Detailed reviews on Big data in Astronomy and Computational Astrophysics are given in Mickaelian [3] and Mickaelian \& Astsatryan [4].

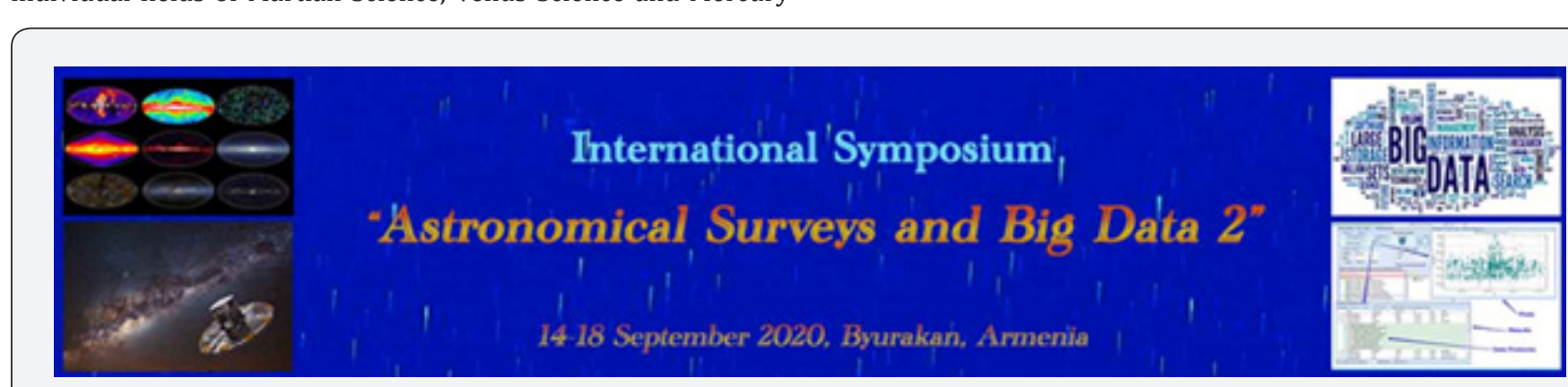

Figure 3: International Symposium “Astronomical Surveys and Big Data 2", where the relation of astronomy to Big Data was discussed. A similar meeting was held in 2015, both in Byurakan, Armenia. 


\section{Astronomy and Space Flights}

The role of celestial mechanics in Space flights and Astronautics is invaluable. The study of the motions of celestial bodies led first to the development of the most classical branch of physics, mechanics, and later to the possibility of controlling the motions of artificial bodies in the near Space, including the launch of many terrestrial artificial satellites, as well as the Astronautics. Moreover, the advantages of astronomy as a super-accurate science are revealed here: the movements of both celestial bodies and artificial satellites and other spacecrafts are calculated with an accuracy of seconds and centimeters. On the other hand, as in the Middle Ages, sailors, and now astronauts use the positions of the stars for navigation. This field is called Astronavigation. With the development of astronautics, reciprocally astronomy progressed rapidly, as most of the electromagnetic waves emitted by space bodies due to the influence of the atmosphere do not reach the Earth's surface. Extra atmospheric or space observatories gave rise to IR, UV, and X-ray astronomy, which greatly expanded our understanding of the Universe. Moreover, even in the optical range, Space Astronomy made significant progress (the example of Hubble Space Telescope is the most characteristic), as both the sensitivity and spatial resolution of the observations were significantly improved.

\section{Astronomy and Meteorology}

In astronomy, the so-called Astroclimate plays an important role in choosing the location of observatories. It is the same climate that is familiar to all meteorologists, but also considering the statistical number of cloudless nights during the year, the degree of atmospheric turbulence, the quality of astronomical images, and a number of other factors that affect astronomical observations. On the other hand, the field of Space Climate is currently developing, which includes the influence of space phenomena and conditions on the Earth and its atmosphere, which is particularly due to the activity of the Sun and the Earth's magnetosphere. Some manifestations of solar activity, such as solar flares and protuberances, dramatically alter the Earth's magnetic field, causing geomagnetic storms that have a serious impact on power plants and all types of communications. Life-threatening ozone holes can also be caused by such influences. Cosmic particles also play an important role, including that from the point of view of the safety of spacecrafts and other space devices. Finally, global warming is also closely linked to Solar activity.

\section{Astronomy, History and Archaeology}

Ancient astronomy or archaeoastronomy (not to be confused with the history of astronomy) studies the manifestations of astronomical knowledge by ancient civilizations, the discovery of historical events through records of astronomical phenomena. These include the ancient observatories, calendars, astronomical rockart, naming of constellations, historical records of astronomical events, Medieval sky maps and astronomical instruments, other items of ancient astronomy and manifestations of the astronomical heritage. There are international organizations related to Archaeoastronomy (IAU commissions and working groups, UNESCO "Astronomy and World Heritage", International Society for Archaeoastronomy and Astronomy in Culture (ISAAC), SEAC, etc.), international meetings are held on this subject and international journals are published also related to Archaeoastronomy (Journal for the History of Astronomy, Mediterranean Archaeology and Archaeometry, Archaeoastronomy, Culture and Cosmos, Journal of Astronomical History and Heritage).

\section{Astronomy and Culture}

Almost in all spheres of culture, such as mythology and religion, folklore, poetry, fine art, architecture, linguistics, symbolism, etc., the influence of celestial phenomena and astronomical understanding have penetrated to one degree or another. Very important is the factor of inspiration by the starry sky, which is a source of many creations, eventually leading to the many manifestations of the celestial bodies and phenomena in art and other spheres of culture. Again, calendars play a significant role in culture and all they are based on astronomical knowledge. Religions and Mythology are tightly related to astronomical knowledge as well. Other manifestations of astronomy in culture are Ethnoastronomy; astronomy in Folklore and Poetry; astronomy in Arts; astronomy in Fashion, Astrolinguistics, Astroheraldry, and Astrosymbolism; Astronomical Observatories as Astronomical Heritage; Astro Tourism and Astronomical Outreach. Figure 4 shows the logo of the meeting "Cultural Astronomy" held in 2016 in Armenia. The two last fields (Archaeostronomy and Astronomy in Culture, AAC) are very closely related and very often they collaborate having joint organizations, holding joint meetings and publishing joint journals. The IAU Working Group Archaeostronomy and Astronomy in Culture (WGAAC) unifies these disciplines as well.

\section{Scientific Tourism, Scientific Journalism, Popular Science, Amateur Astronomy and Astronomical Education}

Astronomical observatories are among the most attractive places for scientific tourism, many observatories in the world have thousands of visitors, which contributes to their recognition, scientific rating, and material benefits. Scientific tourism can be a serious addition to historical and cultural tourism, particularly in Armenia. We have developed a webpage on Astro Tourism for the South West and Central Asia region (http://astrotourism.aras. $\mathrm{am} /$ ), which can be extended to other regions as well. In scientific journalism, according to world statistics, $50-90 \%$ of the topics in different countries are related to the Universe in one way or another: Astronomy, Space Flight, aliens, Archaeostronomy, Astrology, etc. That is why astronomy has a responsible role in the development of scientific journalism. The role of astronomy in the popularization of science is more obvious, as it is by far the most 
attractive science. The IAU also attaches great importance to this, having recently established the Commission \#55 "Communication of Astronomy with the Public". A journal with the same name is published. IAU runs a large-scale astronomy education program, which includes not only school and university astronomy, but also a policy of providing astronomical knowledge to the general population in all age groups.

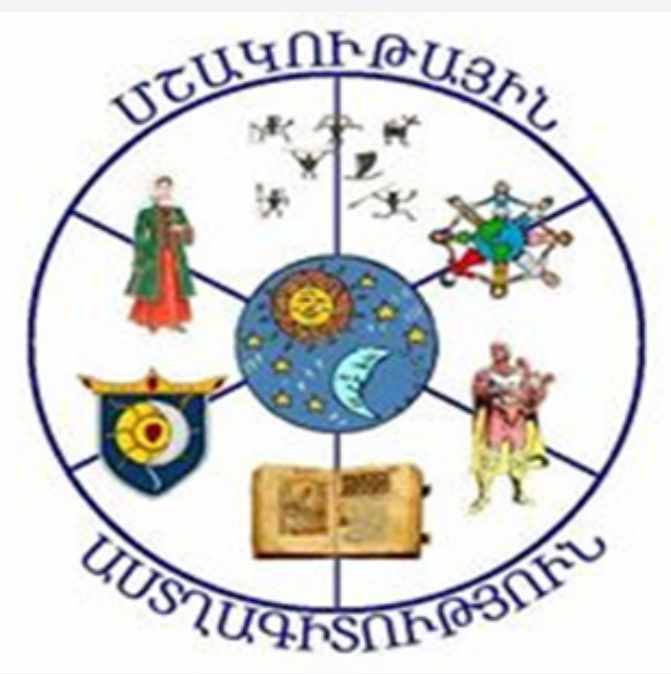

Figure 4: Logo of the meeting "Cultural Astronomy" held in 2016 in Armenia.

\section{Relation of Astronomy to Other Sciences and Culture in Armenia}

In recent years, the Byurakan Astrophysical Observatory and the Armenian Astronomical Society, has taken serious steps to strengthen the connection between astronomy and other sciences and culture. The first steps were the historicalastronomical works of Benik Tumanian, Haik Badalian, Elma Parsamian, Paris Herouni, Grigor Brutean and Karen Tokhatyan (history of Armenian astronomy, calendar, ancient observatories, rock art or petroglyphs). Grigor Gurzadian and Hrant Tovmasian made significant contribution in the field of Space Astronomy. In 2002-2007, Areg Mickaelian digitized the Markarian (the First Byurakan) Survey (FBS, digitized version: DFBS), based on which the Armenian Virtual Observatory [5] was created, the joint result by astronomers and computer specialists. Among the works of Ararat Yeghikian can be distinguished the studies related to Astrochemistry and Astrobiology.

However, a significant step forward was the International Year of Astronomy (IYA-2009) declared by UNESCO, when several international programs were launched to strengthen the cultural connection between astronomy and other sciences. IYA-2009 activities in Armenia were quite wide and multifaceted. ArAS 2009 annual conference on "Astronomy and Society" was held, where the issues of archaeoastronomy, astrobiology, astronomical education, amateur astronomy, astronomy and society were discussed. An archaeoastronomical seminar and conference were organized in 2011 l 2012. Three major events in 2014 [6], 2016 [7] and 2017 [8] were devoted to the Astronomical Heritage and related topics. A detailed review on Armenian activities on AAC is given in Mickaelian \& Farmanyan [9]. In 2010, the group of scientific journalists from Armenia was established by ArAS. The issues of scientific tourism have been discussed since 2009 . With such developments, astronomy can really become a leader in the connection between interdisciplinary and multidisciplinary sciences and science and culture.

\section{The Practical Significance of Astronomy}

The importance of astronomy is reinforced with its practical usage by society. First, it is significant for the formation of person's worldview, especially for the young people. Calendars and Chronology based on astronomical knowledge or the use of astronomical information, Time service, Orientation l. Location/ positioning, Earth l space navigation, optical devices, photography, Celestial Mechanics, Astronautics, further development of Physics and almost all other sciences based on information from Space have played an important role for the development of human society, development of Information Technologies (digital technology, computing methods and the Internet) and many other fields. Computed tomography (CT) and Magnetic Resonance Imaging (MRI) are the excellent examples of the astronomy achievements advancement in other areas. 
A vivid example of the practical significance of astronomy is the use of nuclear energy, which became possible based on astronomical research conducted in the 1930s. In trying to explain the sources of Solar energy, trying to apply all the known forms of energy at the time, scientists were at a dead end, as thousands, millions of times more energy was required than mechanical, thermal, or chemical energy. Finally, Hans Bethe discovered nuclear reactions that release enormous amounts of energy, describing with great precision the processes going on in the Sun's nucleus, the synthesis of 1 nucleus of helium from 4 nuclei of hydrogen. This theory was further developed by Robert Oppenheimer and others, and was later used to build atomic bombs, nuclear power plants, nuclear submarines, and other equipment. Currently, most of Armenia's electricity comes from the Armenian Nuclear Power Plant.

The Role of Astronomy in the Development of Technology

The development of astronomy has been closely related to the development of technology. It was displayed at the measurements and calculations, angle calculating tools, optical devices, photography, photometry, spectroscopy, spectrophotometry, polarimetry, interferometry, adaptive optics, computers, IT sector, digital technology, processing systems, Internet development, e-Science and other fields. All the mentioned fields have both developed astronomy and they themselves have been developed due to the requirements set by astronomy. It is especially evident, the role of astronomy in the creation and development of optical devices, and now it is in the field of IT, because the large flow of information coming from space makes special demands and implies new approaches, in particular, computer clusters grid systems are mainly used by professionals of nuclear physics and astronomers. It can be said that astronomy has been and remains at the front of technology development and perhaps only the military industry with its demands and its role in the development of technology can be compared with astronomy.

\section{The Role of Astronomy in Future Development of Sci-} ences

The role of astronomy in the development of physical sciences, as well as other natural sciences is becoming more and more important due to the use of the vast amount of information coming from the Universe. We would like to mention that the Nobel Prizes are obvious indicator of the increase of the role of Astronomy during the recent years. Several the astronomers or physicists working on astronomical problems have received Nobel Prizes in Physics, which has started in 1901. During the first 100 years, only 6 such teams have been awarded Nobel Prizes. And during 20012020, only 20 years, 6 more "astronomical" teams have also been awarded Nobel Prizes in Physics, including the last two, in 2019 and 2020. We give in Figure 5 the list of "astronomical" Nobel Prizes in Physics.

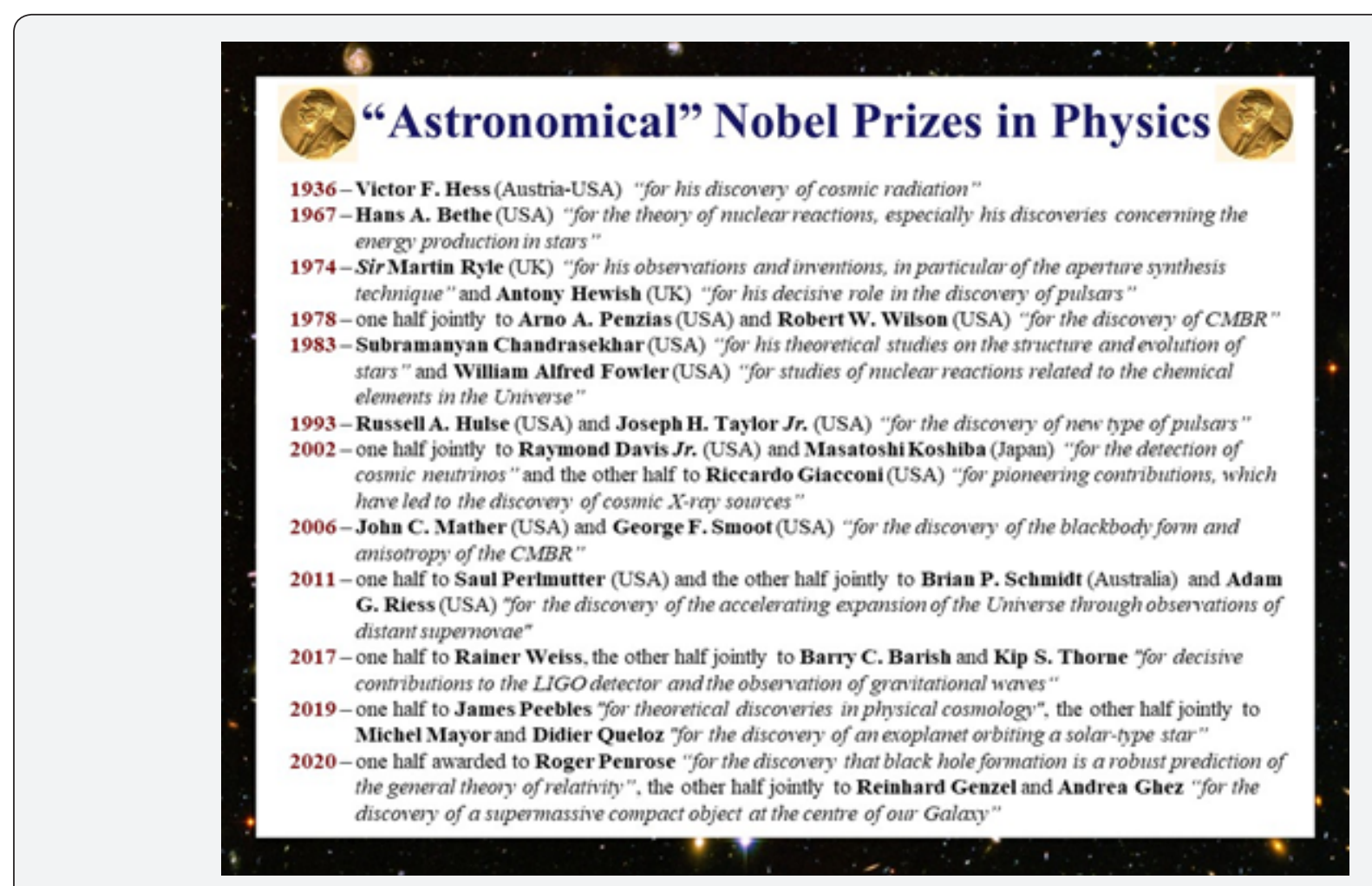

Figure 5: The list of Nobel Prizes in Physics related to astronomical results. 
We have described and analyzed the relation of Astronomy to other (mainly natural) sciences, and we have shown the leading role of Astronomy in the creation of many new inter- and multidisciplinary areas. One can regard several developments of both individual fields of natural sciences, which is already gradually happening and the development of science in general:

a) Establishment and further development of interdisciplinary sciences between individual science disciplines and Astronomy (like Astrophysics, Astrochemistry, Astrobiology, Planetology, etc.)

b) The development of multidisciplinary and transdisciplinary sciences based on involvement of many science disciplines, including Space-based knowledge.

c) Development of Space sciences related to individual science disciplines, such as Communications, Mapping, Meteorology, Remote Sensing, Space Astronomy, Space Biology and Medicine, etc. At present, in fact all explorations, when possible, move to Space and benefit from its possibilities.

d) Further development of Big Data science and computer technologies based on new data coming from the Space and being used for various science fields. Development of e-Science like the creation of Virtual Observatories (VOs) in Astronomy

e) Integration of the scientific data and all information to combined and homogeneous databases, like CODATA and WDS (World Data System) created by the International Science Council (ISC).

\section{References}

1. Doran R, Chaparro G, Farmanyan SV (2020) Overview of IAU OAD Regional Offices and Language Centres, Astronomy in Focus XXX, presented at IAU XXX General Assembly, Vienna, Austria. Proc. of the IAU, pp. 555-557.
2. Mickaelian AM (2015) The Problem of Extraterrestrial Civilizations and Extrasolar Planets, Proc. Conf. Relation of Astronomy to other Sciences, Culture and Society, Eds. Harutyunian HA, Mickaelian AM, Farmanyan SV, NAS RA “Gitutyun”, pp. 83-96.

3. Mickaelian AM (2020) Big Data in Astronomy: Surveys, Catalogs, Databases and Archives. Communications of the Byurakan Astrophysical Observatory (ComBA0) 67: 159-180.

4. Mickaelian AM, Astsatryan HV (2015) Computational Astrophysics. Proc Conf. Relation of Astronomy to other Sciences. Culture and Society, Eds. Harutyunian HA, Mickaelian AM, Farmanyan SV, NAS RA “Gitutyun", pp. 188-201.

5. Mickaelian AM, Astsatryan HV, Knyazyan AV, Mikayelyan GA, Gevorgyan Gh, et al. (2020) The Armenian Virtual Observatory (ArVO). Communications of the Byurakan Astrophysical Observatory (ComBAO) 67(2): 316-322.

6. Harutyunian HA, Mickaelian AM, Farmanyan SV (2015) Relation of Astronomy to other Sciences, Culture and Society. NAS RA "Gitutyun", pp. 460.

7. Farmanyan SV, Suvaryan YuM, Mickaelian AM (2016) Cultural Astronomy in the Armenian Highland. Proc. Young Scientists Conf., held on 20-23 June 2016 in Yerevan, Armenia. (Eds.) Yerevan, "Gitutyun" Publishing House of the National Academy of Sciences of the Republic of Armenia (NAS RA), pp. 250.

8. Farmanyan SV, Mickaelian AM, McKim Malville J, Bagheri M (2019) Astronomical Heritage of the Middle East. Proc. Conf. held 13-17 Nov 2017 in Yerevan, Armenia. (Eds.) San Francisco: Astronomical Society of the Pacific Conference Series (ASP CS), 520: 326.

9. Mickaelian AM, Farmanyan SV (2019) Armenian Archaeoastronomy and Astronomy in Culture, Astronomical Heritage of the Middle East. Proc. Conf. held 13-17 Nov 2017 in Yerevan, Armenia. In: Farmanyan SV, Mickaelian AM, McKim Malville J, Bagheri M. (Eds.). San Francisco: Astronomical Society of the Pacific Conf. Series (ASP CS), 520: 3-13.

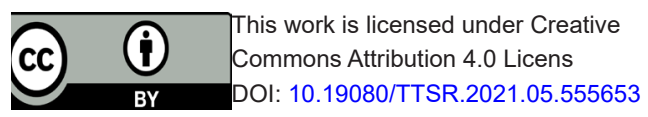

Your next submission with Juniper Publishers will reach you the below assets

- Quality Editorial service

- Swift Peer Review

- Reprints availability

- E-prints Service

- Manuscript Podcast for convenient understanding

- Global attainment for your research

- Manuscript accessibility in different formats ( Pdf, E-pub, Full Text, Audio)

- Unceasing customer service

Track the below URL for one-step submission https://juniperpublishers.com/online-submission.php 